\title{
Evaluation of Mast-ID 15 system for identifying Enterobacteriaceae, some Vibrionaceae, and Acinetobacter
}

\author{
B HOLMES, C A DAWSON \\ From the National Collection of Type Cultures, Central Public Health Laboratory, London
}

SUMMARY Six hundred and twenty one strains (555 Enterobacteriaceae, 46 Vibrionaceae, and 20 Acinetobacter) were examined in the Mast system. The results were consulted in the code book supplied by the manufacturer and those not listed were processed through the manufacturer's full database held on an Apricot microcomputer in our laboratory. The proportion of strains correctly identified was $88 \%$, with $9 \%$ not identified, and $3 \%$ incorrectly identified.

Since our review in $1981^{1}$ of commercially available identification systems, many more have appeared. Some are more advanced technically, such as the Sensititre Auto-ID system in which positive tests yield a fluorescent end product which can then be automatically read. Another approach is to develop a cost effective identification product, such as the Mast-ID system. Several Mast plates are available for the biochemical characterisation of Enterobacteriaceae, Vibrionaceae, and Acinetobacter, used in conjunction with a multipoint inoculator. From this range of plates the manufacturer has selected by computer the 14 which, together with a separate test for motility, effect best differentiation of the 50 taxa chosen for inclusion in the database. These 15 tests comprise the Mast-ID 15 system.

The overall probability of interlaboratory test errors for conventional biochemical tests is about $8 \%$ under routine conditions but probabilities can be as high as $20 \%-30 \%$ for some tests. ${ }^{23}$ Some of these errors are doubtless due to inaccuracies in the making up of the test media. Mast attempt to reduce this problem by providing all the necessary ingredients for a particular test medium, preweighed in sachet form, to be added to the correct amount of water, autoclaved, and dispensed ready for use. The identification plates are designed to complement a similar range of plates for determining antimicrobial susceptibility using the "break-point" approach.

Accepted for publication 11 May 1987
Nineteen strains (or more) are applied to each plate by means of a multipoint inoculator (Mast SCAN 100).

Some 30 plates were introduced by Mast for biochemical characterisation of Enterobacteriaceae. They were used in some laboratories because of their cost effectiveness, but as the manufacturer initially provided no identification scheme such laboratories often devised their own. Clayton et al ${ }^{4}$ selected 14 tests from the Mast range (plus indole and oxidase performed conventionally) by determining the most useful 16 of those contained in a probability matrix, which was based on conventional biochemical tests. ${ }^{5}$ They then examined strains in these 16 tests to build a database derived solely from this source. A profile index was generated and was found to yield rates of correct identification in the range $\mathbf{9 2 - 9 8 \%}$ (the proportion of strains not identified or incorrectly identified was not stated). Funnell et $a l^{6}$ used the same range of Mast plates nominally corresponding to the tests in the API 20E system. They then examined strains in both systems and consulted both sets of results in the API 20E Analytical Profile Index; there was an agreement in identification of $95.4 \%$ to species level and of $\mathbf{9 7 . 7 \%}$ to genus level. Henrichsen and Moyes ${ }^{7}$ selected eight tests from the Mast range (plus indole performed by a paper method), and from an examination of 685 stock cultures constructed a data base and scheme for the identification of Enterobacteriaceae, Acinetobacter spp, and Pseudomonas spp. A separate scheme, based on six tests (including the Mast aesculin test), was devised for the identification of Gram positive organisms. For 438 
field strains examined in both schemes, there was a $91.8 \%$ rate of agreement with identifications obtained by conventional methods.

In this study we evaluated the use of Mast's own scheme in the identification primarily of Enterobacteriaceae, although some members of the Vibrionaceae and some Acinetobacter were also included.

\section{Material and methods}

A total of 621 strains were used in the evaluation (table 1). Most (555) represented 45 Enterobacteriaceae. Of the remaining strains, 46 represented three taxa of Vibrionaceae, 10 were Acinetobacter calcoaceticus and
$10 \mathrm{~A}$ lwoffii. Some were reference strains of the National Collection of Type Cultures (NCTC) and the remainder were field strains referred to the NCTC for identification. All the strains had been previously examined in up to 50 biochemical tests and their identity either confirmed or determined using the methods described by Willcox $e t a l^{8}$ and the probability matrix of Holmes et al. ${ }^{9}$ Some of the strains were referred to the NCTC for identification because they displayed atypical characters but others were referred for other reasons, such as test errors. Where possible, only strains typical in the conventional tests were selected for the evaluation. We aimed to test the system by examining at least 10 to 15 strains (where available) of each of the taxa in the database.

Table 1 Identification of Gram negative fermenters by Mast-ID 15 system

\begin{tabular}{|c|c|c|c|c|}
\hline Taxon & Total No of strains & Correctly identified & Not identified & Incorrectly identified \\
\hline Acinetobacter spp (glucose negative) & 10 & 10 & 0 & 0 \\
\hline Acinetobacter spp (glucose positive) & 10 & 10 & 0 & 0 \\
\hline Aeromonas hydrophila & 18 & 9 & 8 & 1 \\
\hline Citrobacter amalonaticus & 15 & 14 & 1 & 0 \\
\hline Citrobacter diversus/koseri & 15 & 14 & 1 & 0 \\
\hline Citrobacter freundii & 16 & 13 & 2 & 1 \\
\hline Escherichia coli & 10 & 9 & 1 & 0 \\
\hline Edwardsiella tarda & 15 & 12 & 3 & $\mathbf{0}$ \\
\hline Enterobacter agglomerans & 10 & 7 & 2 & 1 \\
\hline Enterobacter cloacae & 13 & 8 & 1 & 4 \\
\hline Enterobacter aerogenes & 10 & 10 & 0 & 0 \\
\hline Enterobacter sakazakii & 16 & 15 & 0 & 1 \\
\hline Hafnia alvei & 15 & 12 & 3 & 0 \\
\hline Kluyvera spp. & 15 & 7 & 6 & 2 \\
\hline Klebsiella pneumoniae/aerogenes & 37 & 33 & 3 & 1 \\
\hline Klebsiella rhinoscleromatis & 16 & 14 & 2 & 0 \\
\hline Klebsiella oxytoca & 15 & 13 & $\overrightarrow{1}$ & 1 \\
\hline Klebsiella ozaenae & 17 & 11 & 6 & 0 \\
\hline Morganella morganii & 12 & 11 & 0 & 1 \\
\hline Morganella morganii Biogroup 1 & 8 & 8 & 0 & $\mathbf{0}$ \\
\hline Plesiomonas shigelloides & 15 & 14 & 1 & 0 \\
\hline Providencia alcalifaciens & 10 & 10 & 0 & 0 \\
\hline Providencia rettgeri & 10 & 10 & 0 & 0 \\
\hline Providencia rustigianii & 15 & 14 & 1 & 0 \\
\hline Providencia stuartii & 10 & 10 & 0 & 0 \\
\hline Proteus mirabilis & 10 & 9 & 1 & 0 \\
\hline Proteus vulgaris & 10 & 10 & 0 & $\mathbf{0}$ \\
\hline Salmonella spp. & 10 & 9 & 1 & 0 \\
\hline Salmonella sub-genus II & 11 & 9 & 2 & 0 \\
\hline Salmonella sub-genus III & 10 & 10 & 0 & 0 \\
\hline Salmonella sub-genus IV & 10 & 9 & 1 & 0 \\
\hline Salmonella choleraesuis & 9 & 9 & 0 & 0 \\
\hline Salmonella gallinarum & 9 & 9 & 0 & 0 \\
\hline Salmonella paratyphi $A$ & 10 & 10 & 0 & 0 \\
\hline Salmonella pullorum & 10 & 10 & 0 & 0 \\
\hline Salmonella typhi & 10 & 10 & 0 & 0 \\
\hline Serratia liquefaciens & 15 & 14 & 1 & 0 \\
\hline Serratia marcescens & 10 & 9 & 0 & 1 \\
\hline Serratia odorifera (Biogroup 1) & 6 & 4 & 1 & 1 \\
\hline Serratia odorifera (Biogroup 2) & 8 & 8 & 0 & 0 \\
\hline Serratia rubidaea/marinorubra & 16 & 13 & 2 & 1 \\
\hline Shigella spp. & 13 & 13 & 0 & 0 \\
\hline Shigella sonnei & 10 & 10 & 0 & 0 \\
\hline Vibrio cholerae & 13 & 13 & 0 & 0 \\
\hline Yersinia enterocolitica & 10 & 8 & 2 & 0 \\
\hline Yersinia frederiksenii & 10 & 10 & 0 & 0 \\
\hline Yersinia intermedia & 15 & 15 & 0 & 0 \\
\hline Yersinia kristensenii & 10 & 10 & 0 & 0 \\
\hline Yersinia pestis & 13 & 13 & 0 & 0 \\
\hline Yersinia pseudotuberculosis & 10 & 4 & 5 & 1 \\
\hline Totals: & 621 & $546(88 \%)$ & $58(9 \%)$ & $17(3 \%)$ \\
\hline
\end{tabular}




\section{TESTS}

The 621 strains were examined in the 15 tests comprising the Mast-ID 15 system (table 2). The system comprised 14 media to be dispensed into Petri dishes and one (motility) to be dispensed into repli dishes (these are $10 \mathrm{~cm}$ square dishes divided into $25 \mathrm{com}$ partments). Overnight bacterial growth was removed from a plate with a straight wire and then suspended in sterile distilled water, sufficient to produce a just visibly turbid suspension. The straight wire was then stabbed into the central point of the motility medium (one strain per compartment). One $\mathrm{ml}$ of the suspension was then dispensed into one of 19 wells in the sterilised polytetrafluoroethylene (PTFE) inoculum block; this was then fitted into the multipoint inculator. A sterile PTFE head fitted with 19 stainless steel inoculating pins was fitted above the block and a stainless steel plate marker (for orientation) was fitted into the head (a template was used to record which pin position corresponded to which culture). The 14 plates were then inoculated. Up to four supplementary plates may also be included: glucose agar, to confirm firstly that the organism is a fermentative one (although $A$ calcoaceticus strains will also give a positive result), but also to facilitate easy recognition of non-fermenters and to use as a positive control in interpreting the carbohydrate reactions; carbohydrate agar base, to use as a negative control in interpreting carbohydrate reactions; $\beta$-glucuronidase agar, for the confirmation of isolates of Escherichia coli and Shi-

Table 2 Tests in Mast-ID 15 system, those chosen by Clayton et al, ${ }^{4}$ and those in API 20 E system

\begin{tabular}{|c|c|c|c|c|}
\hline \multicolumn{2}{|c|}{ Tests } & \multirow{2}{*}{$\begin{array}{l}\text { Tests in } \\
\text { Mast }-I D \\
\text { I5 system }\end{array}$} & \multirow{2}{*}{$\begin{array}{l}\text { Tests chosen } \\
\text { by Clayton } \\
\text { et al }\end{array}$} & \multirow{2}{*}{$\begin{array}{l}\text { Tests in } A P I \\
20 E \text { system }\end{array}$} \\
\hline 1 & Rhamnose & & & \\
\hline 2 & Sucrose & + & + & + \\
\hline 3 & Melibiose & + & & + \\
\hline 4 & Sorbitol & + & & + \\
\hline 5 & Amygdalin & + & & + \\
\hline 6 & Xylose & + & & \\
\hline 7 & Ornithine & + & & + \\
\hline & Phenylalanine & + & + & + \\
\hline & $\begin{array}{l}\beta \text {-Galactosidase } \\
\text { (ONPG) }\end{array}$ & + & + & + \\
\hline 10 & Inositol & + & + & + \\
\hline 11 & Urease & + & + & + \\
\hline 12 & Malonate & + & + & \\
\hline 13 & Motility & + & + & \\
\hline 14 & Indole & + & + & + \\
\hline \multirow[t]{11}{*}{15} & Dulcitol & + & & \\
\hline & Oxidase & & + & + \\
\hline & Gelatin & & + & + \\
\hline & $\mathrm{H}_{2} \mathrm{~S}$ & & + & + \\
\hline & Glucose & & + & + \\
\hline & Mannitol & & + & + \\
\hline & Citrate & & + & + \\
\hline & Lysine & & + & + \\
\hline & Arginine & & & + \\
\hline & Voges-Proskauer & & & + \\
\hline & Arabinose & & & + \\
\hline
\end{tabular}

gella species; and Pseudomonas selective medium, growth on which indicates the test organism is a member of that genus. As no strains of Pseudomonas species were included in the evaluation (as these organisms are not contained in the database), we included only the first three supplementary tests. After the inoculum spots had dried those spots on the malonate and urease plates were isolated by means of a cork borer to prevent excessive diffusion of colour of any positives. After incubation at $37^{\circ} \mathrm{C}$ for $18-24$ hours the results were read with reference to written descriptions of the appearance of positive and negative results. Only the indole test required the addition of a reagent. The results of the 15 basic tests were reduced to a five digit profile number based on octal numbers, ${ }^{1}$ which was then consulted in a profile index. The taxa included in the latter were those considered to be most commonly isolated from clinical specimens. ${ }^{10}$

\section{IDENTIFICATION}

For each profile number in the profile index ("MastID Database" [Codebook]; ours was Issue No 1, dated July 1986), there are listed the two most likely taxa for the given set of test results. For each taxon two values are calculated: the normalised relative likelihood and the modal likelihood fraction. ${ }^{11}$ Only the first value is printed and accompanying it a comment as to the level of the acceptability of the suggested identification (for example, "excellent" if the likelihood is greater than 0.999 ; "acceptable" if the score is above 0.800 but below 0.900 ). Although the second value is not printed, a signal is given in cases where the modal likelihood fraction is the same for both the suggested taxa. Other comments may also be printed beside the appropriate code: "serology", if either Salmonella or Shigella appear as first or second choice for any profile number; "motility at $22^{\circ} \mathrm{C}$ ", if Yersinia enterocolitica or $Y$ pseudotuberculosis should appear as first or second choice for any profile number; and "oxidase" if Aeromonas hydrophila or Plesiomonas shigelloides (but strangely, not Vibrio cholerae) appear as first or second choice for any profile number. Profiles not listed in the profile index could be processed through the full data base supplied by the manufacturer for use on our Apricot microcomputer.

If the identification suggested by the Mast-ID 15 system (relative likelihood $>0.800$ ) agreed with the known identity of the strain then it was taken as correct, and as incorrect if they disagreed. If no definite identification was suggested by the Mast-ID 15 system (relative likelihood $<0.800$ ) then the strain was counted as not identified except where serological confirmation could lead to a correct identification. Conversely, where serology was indicated and a strain would otherwise have been considered as incorrectly 
identified, that identification was counted as not identified. Although we did not perform the serological examination, it was assumed that the results would have either led to correct identifications or refuted the incorrect ones. Where the hazardous pathogens Salmonella typhi, Salmonella paratyphi A, Vibrio cholerae, and Yersinia pestis appear as first or second choice for any profile number, they should be regarded as presumptive identifications until confirmed by a reference laboratory. This rule was followed in the present study and should permit correct identification of all hazardous pathogens.

\section{Results}

The proportion of strains which were correctly identified, not identified, or incorrectly identified are given for each taxon in table 1 . Overall, $88 \%$ of strains were correctly identified, $9 \%$ not identified, and $3 \%$ incorrectly identified. All strains in 22 of the 50 taxa were correctly identified, and misidentifications were confined to 13 of the 50 taxa. Table 3 defines the misidentifications; in one case the discrepancy was only at the biotype level and in six the generic identification was correct but there were discrepancies at the species level.

\section{Discussion}

Many evaluations of commercial identification systems are concerned primarily or exclusively with a comparison of the results obtained in the system with those obtained in the corresponding conventional tests. In an evaluation of the API $20 \mathrm{E}$ system, ${ }^{12}$ where we performed such a comparison, certain tests proved more sensitive in the API system than in conventional media, but for other tests the reverse was true. In conventional media (and the API $20 \mathrm{E}$ system) the decarboxylase tests are carried out anaerobically, with an oil or wax overlay. In the Mast system the decarboxylase tests are performed aerobically so unexpected findings may be obtained-for example, all strains of Acinetobacter lwoffii tested were able to decarboxylate ornithine in the Mast medium but they would not do so conventionally. We therefore consider that any comparison of commercial system results with the results obtained in the corresponding conventional tests has little scientific merit as it is the equivalence of the final identifications that is important. Clayton et al, ${ }^{4}$ however, based their initial test selection on a matrix compiled from conventional tests, and then compiled a database from purely Mast test results (with the indole and oxidase tests performed conventionally) and improved it using various statistical analyses. Their initial choice of tests, however, cannot have been as ideal as that compiled by the manufacturer from a database derived purely from testing strains in all 30 Mast plates and then selecting the most useful of those. The choice of tests will also vary according to the taxa included in the database and more non-fermenters were included in the scheme of Clayton et $a l^{4}$ than in the manufacturer's own scheme. Nevertheless, both the MastID 15 identification scheme, that of Clayton et al, ${ }^{4}$ as well as that for the API $20 \mathrm{E}$ system, are aimed primarily at the Enterobacteriaceae, so some similarity in the tests included in all three systems might be expected (although as far as we know the choice of tests to include in the API 20E system was not made by examining strains in a wider range of tests in API $20 \mathrm{E}$ format and then selecting the most differential of those). The tests included in the Mast-ID 15 system, in that of Clayton $e t a l^{4}$ and in the API 20E system, are listed in table 2 . The choice of tests used in the two systems based on Mast media varies considerably, although both systems include malonate and motility, and the Mast-ID 15 system includes dulcitol and xylose; none of these tests appear in the API 20E system. Conversely, arabinose, arginine, and VogesProskauer are the only tests included in the API 20E system but not in either of the other two systems. The identification scheme of Henrichsen and Moyes ${ }^{7}$ has not been discussed. As that scheme was designed for the identification of isolates directly from urine, it is not directly comparable with the other schemes considered here.

Our overall rate of correct identification of $88 \%$ is lower than that obtained by Clayton et $a^{4}$ and Funnell et al. ${ }^{6}$ There are two probable explanations for this. Firstly, we used entirely stock cultures rather than fresh clinical isolates. Although we subcultured each strain several times before testing it in the MastID 15 system, some may not have achieved normal levels of enzymatic activity. Secondly, we examined roughly equal numbers of all 50 taxa in the database, including hazardous pathogens; in the routine laboratory the proportion of strains of each species tested generally varies with the distribution of the species in the incoming specimens. Clayton et $\mathrm{l}^{4}$ included 41 taxa in their database, but only 25 of those were members of the Enterobacteriaceae, four belonged to the Vibrionaceae, and 10 were non-fermenters (the two remaining taxa were Chromobacterium violaceum and Pasteurella sp). Funnell et $a l^{6}$ included only 16 taxa in their evaluation; 15 were named species of Enterobacteriaceae and one was a miscellaneous category. For a general comparison only (as not all the same strains were examined in each study) in the API $20 \mathrm{E}$ system (comprising 21 tests), we obtained a rate of correct identification of $88 \%$ with $10 \%$ not identified and $2 \%$ incorrectly identified. ${ }^{12}$ Even with probability matrices based on 50 or so conventional tests, 
the rates of correct identification reported are of a similar order: $90 \cdot 3 \%,{ }^{5} 89 \cdot 2 \%,{ }^{9}$ although with rates of incorrect identification close to, or at, $0 \%$. Given the cost effectiveness of the Mast-ID 15 system it is perhaps unfortunate that the number of tests was not equal to or greater than that in the API $20 \mathrm{E}$ system, which should improve correct and incorrect identification rates. Clayton et $a l,{ }^{4}$ however, also considered a similarly small number of tests to be adequate.

The identification rates on the Mast-ID system were good compared with API 20E and conventional methods, although it should be pointed out that some of the strains used to compile the database were also used to evaluate it. This has, however, been the practice in other evaluations and occurs particularly among the rarer taxa where so few strains are available. ${ }^{9}$ The Mast-ID 15 system was not perhaps quite as sophisticated as other systems. Identification to genus level only was probably more common than with the API 20E system. The Mast-ID 15 system could not differentiate adequately between Providencia alcalifaciens and $P$ rustigianii, and isolates identified only to genus level; nor between Shigella species (other than $S$ sonnei) and Yersinia pestis, but in this case "serology" is signalled and the latter organism is to be considered as presumptively identified until confirmed by a reference laboratory, and so a final identification is possible using the system. Discrimination between Yersinia frederiksenii and $Y$ intermedia was also inadequate, and although all strains were identified correctly, this was in most cases only to genus level. There was also inadequate discrimination between Acinetobacter spp (glucose positive) and Salmonella typhi and between Acinetobacter spp (glucose negative) and Salmonella pullorum, but again, as serology is required a definite identification could be achieved in both cases. In the latter case the result of the glucose supplementary test will also indicate the correct identification. There was inadequate differentiation between Enterobacter cloacae and several other taxa (Enterobacter aerogenes, E sakazakii, and Kluyvera spp) as shown by the higher rate of incorrect identification obtained for this species. The manufacturer informed us that the problem with this taxon is that different strains give different results in so many of the chosen tests. We consider that the problem of incorrect identifications of $E$ cloacae is the major problem for the manufacturer to resolve in the Mast-ID database. In the evaluation of the probability matrix of Holmes et al ${ }^{9}$ Enterobacter cloacae also had one of the lowest identification rates. Other improvements could include the use of supplementary tests to further the identification in cases where discrimination on the basic tests is inadequate, which may improve identification rates, particularly for the following taxa with lower rates of correct identification: Aeromonas hydrophila, Enterobacter agglomerans, Enterobacter cloacae, Kluyvera spp, Klebsiella ozaenae, Serratia odorifera (biogroup 1), and Yersinia pseudotuberculosis. Otherwise, some taxa could perhaps be combined, such as $P$ alcalifaciens and $P$ rustigianii, $Y$ frederiksenii and $Y$ intermedia. Clayton $e^{2}$ al ${ }^{4}$ also had to combine certain pairs of taxa where the discrimination between them was inadequate. At present, if the relative likelihood of the taxon with the highest value is $<0.800$ but the second taxon listed belongs to the same genus as the first, then the strain is considered to have been identified to genus level. If, however, identification to genus level was allowed only when the sum of the scores of the first two taxa listed (provided they belong to the same genus) was $>0.800$, then this might reduce the misidentification rate (such a rule would have prevented three of the misidentifications observed in this study) (table 3). Another major improvement might be to use the modal likelihood fractions as an identification statistic by calculating the ratios between these values for each of the most likely taxa suggested.

The major technical problems encountered with the

Table 3 Misidentification of strains by Mast-ID 15 system

\begin{tabular}{|c|c|c|}
\hline \multicolumn{2}{|c|}{ Organism } & \multirow{2}{*}{$\begin{array}{c}\text { Incorrectly identified as: } \\
\begin{array}{c}\text { Yersinia enterocolitica/ } \\
\text { Yersinia pestis }\end{array}\end{array}$} \\
\hline 1 & Aeromonas hydrophila & \\
\hline 2 & Citrobacter freundii & Serratia odorifera \\
\hline 3 & Enterobacter agglomerans & Klebsiella rhinoscleromatis \\
\hline 4 & Enterobacter cloacae & Enterobacter aerogenes \\
\hline 5 & Enterobacter cloacae & Kluyvera spp \\
\hline 6 & Enterobacter cloacae & Enterobacter aerogenes \\
\hline 7 & Enterobacter cloacae & Enterobacter sakazakii \\
\hline 8 & Enterobacter sakazakii & Enterobacter agglomerans \\
\hline 9 & Kluyvera spp & $\begin{array}{l}\text { Yersinia frederiksenii| } \\
\quad \text { Yersinia intermedia*† }\end{array}$ \\
\hline 10 & Kluyvera spp & Citrobacter freundii \\
\hline 11 & $\begin{array}{l}\text { Klebsiella pneumoniae/ } \\
\text { aerogenes }\end{array}$ & Enterobacter agglomerans \\
\hline 12 & Klebsiella oxytoca & $\begin{array}{l}\text { Klebsiella pneumoniae/ } \\
\text { aerogenes }\end{array}$ \\
\hline 13 & Morganella morganii & $\begin{array}{l}\text { Morganella morganii } \\
\text { Biogroup } 1\end{array}$ \\
\hline 14 & Serratia marcescens & Serratia liquefaciens \\
\hline 15 & $\begin{array}{l}\text { Serratia odorifera } \\
\text { (Biogroup 1) }\end{array}$ & $\begin{array}{l}\text { Enterobacter cloacae/ } \\
\quad \text { Enterobacter aerogenes }{ }^{*} \dagger\end{array}$ \\
\hline 16 & $\begin{array}{l}\text { Serratia rubidaeal } \\
\text { marinorubra }\end{array}$ & $\begin{array}{l}\text { Enterobacter cloacael } \\
\quad \text { Enterobacter aerogenes* } \dagger\end{array}$ \\
\hline 17 & $\begin{array}{l}\text { Yersinia } \\
\quad \text { pseudotuberculosis }\end{array}$ & $\begin{array}{l}\text { Acinetobacter spp } \\
\quad \text { (glucose positive) }\end{array}$ \\
\hline
\end{tabular}

*Identification to genus level only. + Sum of relative likelihoods of these two taxa $<0.800$.

Three strains of Shigella species (other than $S$ sonnei) reached identification level to Yersinia pestis and one strain of Vibrio cholerae reached identification level to Aeromonas hydrophila (although with $V$ cholerae as second choice). These were not counted as misidentifications in this study as the application of the rules concerning serology or presumptive identification of hazardous pathogens, or both, would lead to a correct identification in each case. 
Mast-ID 15 system were in the reading of certain of the tests. The use of the carbohydrate base, as a negative control, can be definitely recommended to aid in interpreting weak or doubtful carbohydrate reactions. The glucose plate as a positive control may also prove useful. Others, particularly indole, needed practice at reading. Positive and negative control strains for certain tests can be included on each plate, and the manufacturer might consider recommending particular culture collection strains to be used for this purpose. Break-point susceptibility plates, also available from Mast, can be read automatically by the Mastascan, which contains a black and white television camera. A similar instrument with a colour TV camera is apparently being developed which will automatically read both break-point susceptibility and ID plates. This may overcome the difficulties of reading some of the ID tests. Mucoid klebsiella strains often failed to grow sufficiently on the Mast-ID 15 plates. Presumably, an apparently adequate inoculum contains insufficient bacterial cells, and in this study a very dense suspension proved necessary if satisfactory growth was to be obtained.

Although we did not carry out a costing exercise ourselves, Clayton et $\mathrm{al}^{4}$ found the cost per identification by Mast-ID to be about $20 \%$ of that by API 20E (assuming a set of 18 strains is tested in the Mast system). Funnell et $a l^{6}$ determined a comparable figure of around $12 \cdot 5 \%$.

We thank E Farrokhi and S Odusoga for technical assistance.
References

1 D'Amato RF, Holmes B, Bottone EJ. The systems approach to diagnostic microbiology. CRC Crit Rev Microbiol 1981;9:1-44.

2 Lapage SP, Bascomb S, Willcox WR, Curtis MA. Identification of bacteria by computer: general aspects and perspectives. $J$ Gen Microbiol 1973;77:273-90.

3 Sneath PHA. Test reproducibility in relation to identification. Int J Syst Bacteriol 1974;24:508-23.

4 Clayton P, Feltham RKA, Mitchell CJ, Sneath PHA. Constructing a data-base for low cost identification of Gram negative rods in clinical laboratories. J Clin Pathol 1986;39:798-802.

5 Bascomb S, Lapage SP, Willcox WR, Curtis MA. Identification of bacteria by computer: identification of reference strains. $J$ Gen Microbiol 1973;77:291-315.

6 Funnell GR, Parkinson DL, Bradbury R. Biochemical identification of enterobacteriaceae by multipoint inoculation using Mast ID media. Aust J Med Lab Sci 1986;7:75-7.

7 Henrichsen C, Moyes A. A semi-automated method for the culture, identification and susceptibility testing of bacteria direct from urine specimens. Med Lab Sci 1987;44:50-8.

8 Willcox WR, Lapage SP, Holmes B. A review of numerical methods in bacterial identification. Antonie Van Leeuwenhoek 1980;46:233-99.

9 Holmes B, Dawson CA, Pinning CA. A revised probability matrix for the identification of Gram-negative, aerobic, rodshaped, fermentative bacteria. $J$ Gen Microbiol 1986;132:113-35.

10 Farmer JJ III, Davis BR, Hickman-Brenner FW, et al. Biochemical identification of new species and biogroups of Enterobacteriaceae isolated from clinical specimens. J Clin Microbiol 1985;21:46-76.

11 Dybowski W, Franklin DA. Conditional probability and the identification of bacteria: a pilot study. J Gen Microbiol 1968;54:215-29.

12 Holmes B, Willcox WR, Lapage SP. Identification of Enterobacteriaceae by the API $20 \mathrm{E}$ system. J Clin Pathol 1978;31:22-30.

Requests for reprints to: Dr B Holmes, NCTC, Central Public Health Laboratory, 61 Colindale Avenue, London NW9 5HT, England. 